\title{
The Study of Mode Frequency Updating Parameters Selecting Method
}

\author{
Fe-Li QIU ${ }^{1, a}$, Li-Min ZHANG ${ }^{1, b}$, Wei-Hua ZHANG ${ }^{1}$, Ai-Qin TIAN ${ }^{2}$ \\ ${ }^{1}$ Traction State Key Laboratory, Southwest Jiaotong University, Chengdu 610031, China \\ ${ }^{2}$ CSR Sifang Co.Ltd, Qingdao Shandong 266000, China \\ afl_43@163.com, b zhang-Im01@163.com
}

Keywords: Model Updating, Parameters Selection, Sensitivity Analysis, Significance Analysis.

\begin{abstract}
Parameters selection plays an important role in the model updating and a good parameters selection improves the efficiency of model updating. The sensitivity and the significance method are widely used for parameters selection. The theory and application are researched. A real structure was selected to research the parameters selection. The result shows these two methods can all be used in the simple structure and the significant method is more convenient for use in large finite element model. However, a new experiment design method is required to reduce the sampling space for significance method using in too many parameters condition.
\end{abstract}

\section{Introduction}

The traditional parameters selecting methods are mainly based on the experience of engineers in the process. It's affected heavily by the subjective factors [1], and there is no numerical standard for parameters which should be selected or not. Many methods of parameters selecting were researched in the past years. The sensitivity analysis and significance analysis methods were used widely for the parameters selecting. The sensitivity analysis method is based on the structure responses partial differential to the parameters, and the partial differential values can show the sensitivities of structure responses with the parameter changes [2]. As the parameters changing, the statistic method was used to calculate the effects on the structure response in the significance analysis. Firstly, the sampling point's responses should be computed in the sampling space [3-5].

Comparing the two methods, the theory of the sensitivity analysis method is easy to understand, and the updated parameters have clear physical sense[6]. However, the differential effects between various parameters were not considered as the partial differential just at one certain point which may cause the objective function optimization in local range. In the other hand, the significance analysis method gives the effects of responses in the whole variables space which is more sensible than the sensitivity method [7].

In the background above, an experimental structure was selected to research the differences of the two methods in the theory and application fields. Choosing the modal frequencies as the objectives, the best application conditions were pointed out in order to update the finite element model.

\section{Theory of Parameter Selecting}

\section{Sensitivity Analysis}

There are two ways to get the sensitivity matrix. One is the direct derivation method by exact system equations, the other one is the finite difference method.

Direct Derivation Method. Based on the vibration theory, the eigen-equations can be written as the follows:

$$
\left([K]-f_{r}[M]\right)\left\{\phi^{(r)}\right\}=\{0\}
$$

$[K]$ and $[M]$ are the represent the stiffness matrix and mass matrix of the structure, $f_{r}$ is the $r t h$ eigenvalue, $\left\{\phi^{(r)}\right\}$ is the $r$ th mode shape. 
As the orthogonality of the eigenvectors, the mode shapes can be normalized by the mass matrix.

$$
\left\{\phi^{(r)}\right\}^{T}[M]\left\{\phi^{(r)}\right\}=I
$$

The derivation of the Eq. 1 and Eq. 2 were calculated, the following formulas can be gotten.

$$
\begin{aligned}
& \left([K]-f_{r}[M]\right) \frac{\partial\left\{\phi^{(r)}\right\}}{\partial p_{i}}+\left(\frac{\partial[K]}{\partial p_{i}}-\frac{\partial f_{r}}{\partial p_{i}}[M]-f_{r} \frac{\partial[M]}{\partial p_{i}}\right)\left\{\phi^{(r)}\right\}=0 \\
& \left(\frac{\partial\left\{\phi^{(r)}\right\}}{\partial p_{i}}\right)^{T}[M]\left\{\phi^{(r)}\right\}+\left\{\phi^{(r)}\right\}^{T}[M]\left(\frac{\partial\left\{\phi^{(r)}\right\}}{\partial p_{i}}\right)+\left\{\phi^{r}\right\}^{T} \frac{\partial[M]}{\partial p_{i}}\left\{\phi^{(r)}\right\}=\{0\}
\end{aligned}
$$

The first order sensitivity of frequencies to the parameters can be archived with the symmetric of the stiffness matrix and mass matrix[8-9].

$$
\frac{\partial\left\{f^{(r)}\right\}}{\partial p_{i}}=\left\{\phi^{(r)}\right\}^{T}\left(\frac{\partial[K]}{\partial p_{i}}-f_{r} \frac{\partial[M]}{\partial p_{i}}\right)\left\{\phi^{(r)}\right\}
$$

The direct derivate method with the vibration equations is applicative in the discrete system or simple continuous structure. In the complex and large structure, it's really hard to formulate all the equations which will lead the direct derivate method invalid.

Finite Difference Method. Using $p$ to represent the design parameters of the structure, the mode frequency vectors $\{f\}$ can be defined as the implicit function of parameters $p$. With the parameters slight changes, the first order Taylor series expansion of the $r$ th mode frequency $\left\{f^{(r)}\right\}$ can written as the follow formula[10]:

$$
\left\{f^{(r)}(p+\Delta p)\right\}=\left\{f^{(r)}(p)\right\}+\sum_{i=1}^{n} \frac{\partial\left\{f^{(r)}\right\}}{\partial\left(p_{i}\right)} \Delta p_{i}
$$

As the large number of finite element model, it's difficult to get the partial difference of the mass and stiffness matrix. The first order finite difference sensitivity of natural frequency can be calculated by the ANSYS software, as shown in the Eq.7.

$$
S_{i, j}(f, p)=\frac{\left\{f_{i}^{(r)}\left(p_{j}+\Delta p_{j}\right)\right\}-\left\{f_{i}^{(r)}\left(p_{j}\right)\right\}}{\Delta p_{j}}
$$

The matrix form of the parameter sensitivity is shown in the Eq. 8 .

$$
[S]=\left[\begin{array}{ccc}
\frac{\partial f_{1}}{\partial p_{1}} & \cdots & \frac{\partial f_{1}}{\partial p_{n}} \\
\vdots & \ddots & \vdots \\
\frac{\partial f_{\mathrm{m}}}{\partial p_{1}} & \cdots & \frac{\partial f_{m}}{\partial p_{n}}
\end{array}\right]
$$

$n$ is the number of the parameters which are need to be updated, $m$ is the number of selected eigenvalue.

\section{Significance Analysis}

The experiment design must be executed before the significance analysis.

Experiment Design. The central composite design is the most widely used in various sampling space design method [11-13]. Firstly, $2^{m}$ of sampling points were constructed by the orthogonal tab. Only one point experiment needed in the center position $(0,0 \ldots 0)$, as no uncertain factor in numerical simulation. Then, two symmetric points were selected as sampling points in each factor 
axis with $\pm a$ distances; there are $2 m$ points selected with $m$ factors [14].

The total number of central composite design sampling points is :

$$
\mathrm{N}=2 \mathrm{~m}+1+2 \mathrm{~m}
$$

Significance Analysis. The F-inspection method of statistic was used for significance analysis. The parameters which affect heavily on the structure response were selected with the F-value hypotheses testing. The calculating method of F-value based on statistic is shown in the Eq. 10 [15].

$$
F=\frac{\operatorname{SSE}\left(x_{1}, x_{2}, \ldots, x_{m-1}\right)-\operatorname{SSE}\left(x_{1}, x_{2}, . ., x_{m}\right)}{\operatorname{SSE}\left(x_{1}, x_{2}, . ., x_{m}\right) /(k-m-1)}
$$

$\operatorname{SSE}\left(x_{1}, x_{2}, \ldots, x_{m-1}\right)$ is the error square of regression model which concludes $m-1$ independent variables, while $\operatorname{SSE}\left(x_{1}, x_{2}, . ., x_{m}\right)$ is the error square of regression model which concludes $m$ independent variables, $k$ is the number of the independent variables in the regression model.

If the significance level is selected as $\partial$,then:

$$
P\left\{F_{j} \geq F_{1-\partial}(1, n-m-1)\right\}=\partial
$$

$F_{j}$ can be calculated from the Eq. 10, $F_{1-\partial}$ represents the critical value of $F$ hypotheses, (1, $n-m$-1)represent single variable and $n-m-1$ freedom, $P$ is the possibility.

The variables have important effect on the response when $\mathrm{P} \leq \partial$. In opposite, the variables are not significant to the response as $\mathrm{P} \geq \partial$.

\section{The Application of Parameters Selecting}

\section{Sensitivity Analysis}

The geometry model and the numbering components of frame structure are all shown in the Fig.1.

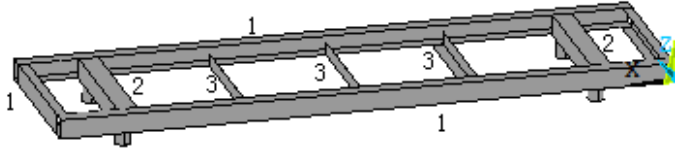

Fig. 1 The Geometry Model and Components of the Frame

The initial parameters of the frame finite element model are shown in the table1.

Tab.1 The Initial Parameters of the Frame Model

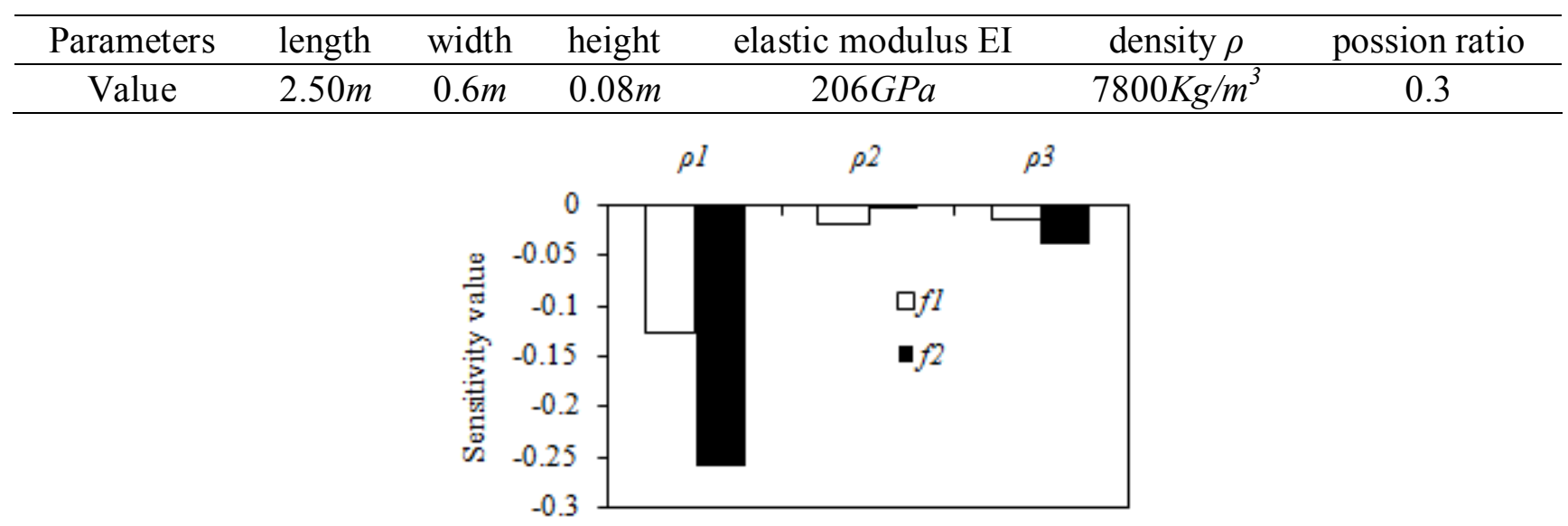

Fig. 2 The Frequency Sensitivity of the Density 


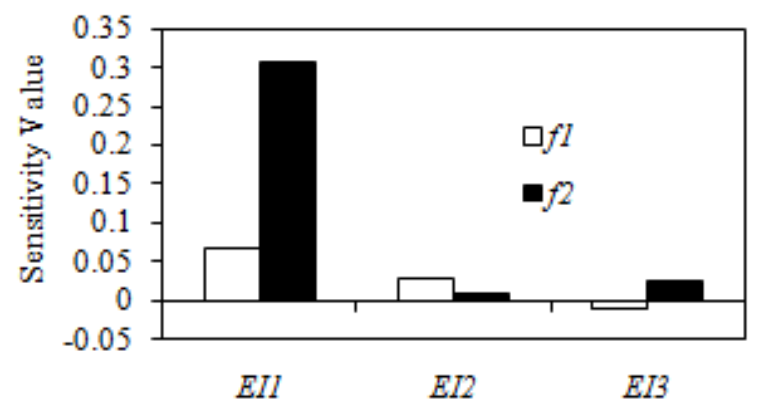

Fig. 3 The Frequency Sensitivity of the Elastic Modulus

There are three components composed the frame structure in the figurel and its geometry dimension is accurate relatively. The elastic modulus $\mathrm{E}_{i}$ and density $\rho_{i}$ of the frames were selected as six parameters to be updated. In order to avoid the aimless and improve the efficiency, the sensitivity analysis was executed to provide the advice for updating parameters selecting [9].

The first and second mode frequency sensitivities to the density and elastic modulus were calculated with the $1 \%$ increase on the initial of the density and modulus value. The results are shown in the Fig.2 and Fig.3.

In the Fig.2, the first order frequency is sensitive to the density $\rho_{1}$ and the second order frequency is sensitive to the density parameter $\rho_{1}$ and $\rho_{3}$. In Fig.3, it's shown that the first and second frequencies are all sensitive to the elastic modulus $\mathrm{EI}_{1}$. With the sensitivity result, the parameters E1, $\rho_{1}$ and $\rho_{3}$ were selected as the updating parameter of the model.

\section{Significance Analysis}

In the significance analysis, the elastic modulus (EI1, EI2 and EI3) and density ( $\rho 1, \rho 2$ and $\rho 3)$ of the three components are selected as updating parameters. As the little error of density, the range 1 $\pm 5 \%$ is defined as the density value range. The elastic modulus value range is $1 \pm 20 \%$ while it may have large error existent. The range of parameters' value is shown in Tab.2.

Tab.2 The Range of Elastic Modulus and Density

\begin{tabular}{ccccccc}
\hline parameters & EI1 & EI2 & EI3 & $\rho 1$ & $\rho 2$ & $\rho 3$ \\
\hline min values & 1.648 & 1.648 & 1.648 & 7.41 & 7.41 & 7.41 \\
middle values & 2.06 & 2.06 & 2.06 & 7.8 & 7.8 & 7.8 \\
max values & 2.472 & 2.472 & 2.472 & 8.19 & 8.19 & 8.19 \\
\hline
\end{tabular}

The unit of the symbol $\mathrm{EI}_{\mathrm{i}}$ and $\rho_{\mathrm{i}}$ are Gpa and $10^{3} \mathrm{~kg} / \mathrm{m}^{3}$ separately in Tab.5.

The experiment sample was designed with six parameters based on the central composite method. The values of the sample were calculated in the finite element model and the sample points are shown in the Tab.3.

Tab.3 The Contents of Sample Points

\begin{tabular}{cc}
\hline Sample space & Number of the sample \\
\hline$( \pm 1, \pm 1, \pm 1, \pm 1, \pm 1, \pm 1)$ & $2^{6}$ \\
$(0,0,0,0,0,0)$ & 1 \\
$( \pm 1,0, \ldots, 0) \sim(0,0, \ldots, \pm 1)$ & $2 \times 6$ \\
\hline
\end{tabular}

In Tab.3, values $\#$ represent the min value and the max value of the parameters and zero is the middle value of parameter which can be found in the Tab.2. There are 77 sample points and sample values which are not shown in the papers due to space limitations

The parameters and its interference significance is studied with the 77 sample data gotten by experimental design. The significances of first and second order frequency $(f 1$ and $f 2)$ are shown in the Fig.3. 


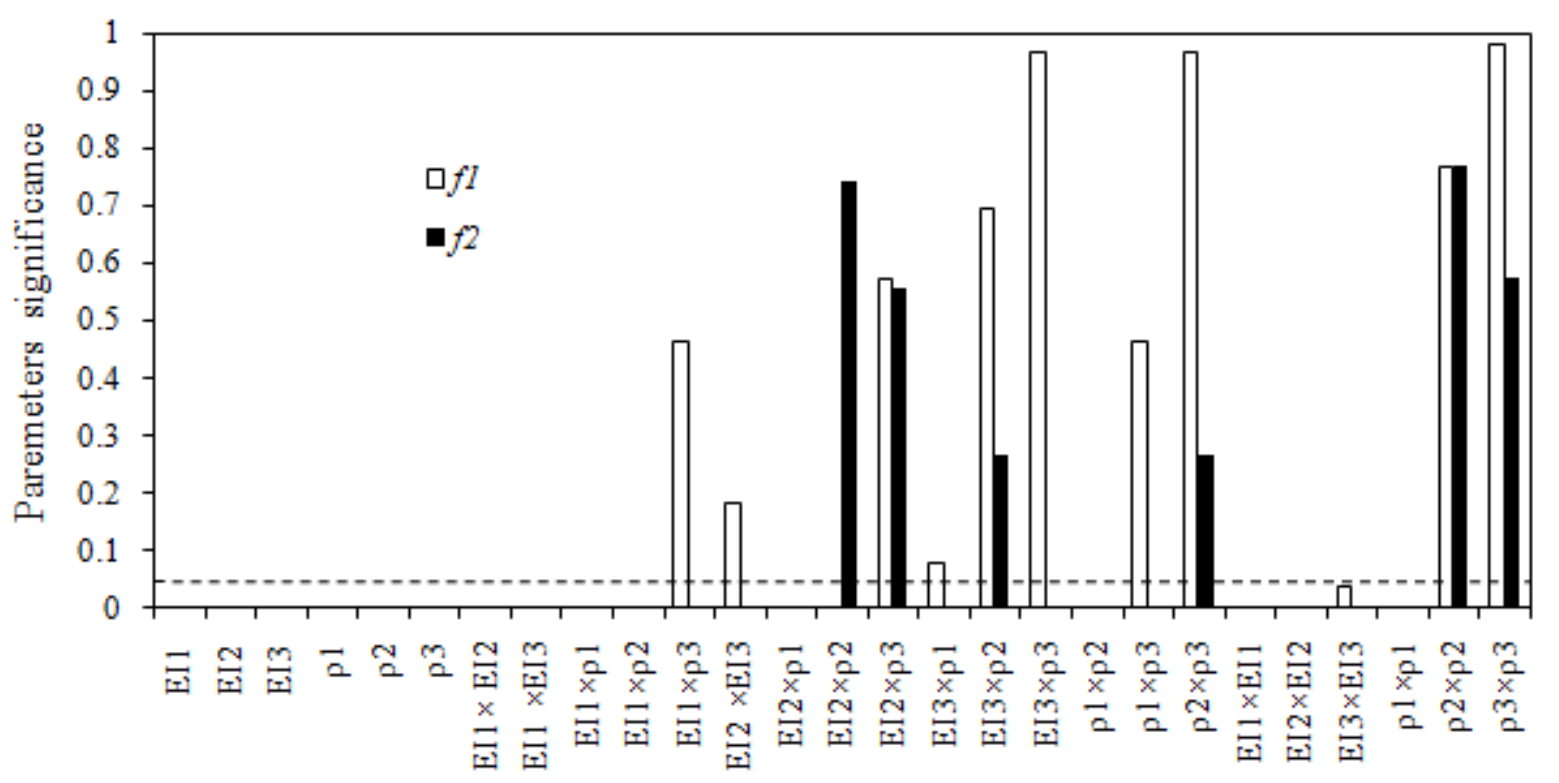

Fig. 4 The Significance of Parameters

In the Fig.4, the literal dashed line location is $a=0.05$. The elastic modulus (EI1,EI2,EI3) and density $(\rho 1, \rho 2, \rho 3)$ parameters are significant to the first and second natural mode frequencies $(f 1$ and f2). At the same time, it can be seen that some interference parts are not significant to the frequencies. The efficiency can be improved with the insignificant parts removed.

\section{Conclusions}

The sensitivity method based on the Tayor series expansion has the advantages of simple principle and high operability. But the sensitivity matrix is calculated in the certain value point which needs to be calculated repeatedly in the update process. The sensitivity analysis will waste lost of time in large finite element model with too much iterations. Comparing with the sensitivity method, the significance method based on the statistics in the full parameters space is more sensible for parameters select. The interferences between parameters were also taken into consideration in the significance analysis. The significance method is available in the large structure model without the need of repetition. However, too many parameters will lead a large number of sample space points and it's important to use a new experiment design.

\section{References}

[1]Lan Hao-lun. Stiffness Sensitivity Analysis for the stainless steel subway vehicle's Carbody,J.Da lian jiaotong university.2011.

[2]Yuan Ai-ming Studies on Several Key Problems of Finite Element Model Updating Based on the Sensitiviyt Analysis ,D.Nanjing: Southeast university.2006.

[3]Guo Qing-tao, Zhang Ling-mi, Fei Qing-guo.From fe model updating to model validation adcances in modeling of dynamic structures ,J. Advances in mechanics. 36(2006)36-42.

[4]Gao Min-lin.Finite element model validation of the continuous rigid frame bridge based on structural health monitoring,D.Fuzhou: Fuzhou university, 2008.

[5]Lin Xiao-song. Finite element model validation of the bridge based on the higher orders response surface method,D. Fuzhou:Fuzhou university, 2010.

[6]Qiu Fe-li, Zhang Li-min, Zhang Wei-hua. Study on frame model updating based on response surface method,J. Noise and vibration and control, 34(2014)139-143. 
[7]QIU Fei-li, ZHANG Li-min, ZHANG Wei-hua, ZHANG Yuan-liang. Updating and Optimization of Design Parameters in Frame Structure Modeling, J. Noise and vibration and control,34(2014)36-40.

[8]Wang Shu-ting, Wang Ting, Lai Fu-kun. The mode and mode sensitivity analysis of the carbody structure, J.Light Vehicles,5(2006)4-7.

[9]QU Xiao- bin,HE Zhi-gang, ZHANG Min-zhong, YU Cheng- xiang. Sensitivity analys is and optimization for city bus body, J.Machinery Design \& Manufacture,1(2007)79-80.

[10]Dai Hang, Yuan Ai-min.Structure model updating based on sensitivity analysis. The science press, Beijing, 2011.

[11]Douglas C. Montgomery. Design and analysis of experiments. China Statistics Press, Beijing, 1998.

[12]Xi Guang, Wang Zhi-heng, Wang Shang-jing. Aero dynamic optimization design of turbo machinery with approximation model method,J. Journal of xi-an jiaotong university, 2(2007)125-135.

[13]Zhao Zu-de, Chen Xue-wen,Chen Jun. The design optimization for hot forging process of connecting-rod based on approximate model and numerical simulation, J. Journal of shanghai jiaotong university,5(2008)758-751.

[14]WU Jia-yan, LI Hao-lin. FEM parameters calibration for thermal characteristics of CNC machine spindle system based on RSM,J. Machine Design and Research,26(2010)21-24

[15]Zong Zhou-hong,Ren Wei-xin. Finite element model updating and model validation of bridge structure. China Communication Press, BeiJing,2012. 